\title{
Desafíos Emergentes en la Nutrición del Adolescente
}

\author{
M. LUISA AGUIRRE C. ${ }^{1}$, CARLOS CASTILLO D. ${ }^{2}$, CATALINA LE ROY O. ${ }^{2}$ \\ 1. Departamento de Pediatría y Cirugía Infantil Campus Oriente Facultad de Medicina Universidad de Chile. \\ Hospital Luis Calvo Mackenna. \\ 2. Departamento de Pediatría y Cirugía Infantil Campus Centro Facultad de Medicina Universidad de Chile. \\ Hospital San Borja-Arriarán.
}

\begin{abstract}
Emergent Challenges in Adolescent Nutrition

Adolescence is a dynamic period of the life cycle, with significant physical, psychological and social changes, leading to autonomy and independence in eating behaviors. During this time there is an increased risk in nutritional behavior, including excessive intake of calories, increased consumption of fat, cholesterol, and simple carbohydrates, and nutrient deficiencies such as calcium. In addition other behaviors are at risk, including disorganized mealtimes, eating outside the home, and the onset of eating behavior disorders and addictions (caffeine, alcohol). It is necessary to develop preventive educational programs including physical activity among adolescents, to promote healthy behaviors into adulthood. Significant literature in Chile and South America is reviewed.
\end{abstract}

(Key words: Nutrition, adolescents, diet, adiccions, nutritional behavior).

Rev Chil Pediatr 2010; 81 (6): 488-497

\section{RESUMEN}

La adolescencia es un período dinámico del ciclo vital, con grandes cambios físicos, psicológicos y sociales, los que conducen a autonomía e independencia en la conducta alimentaria de los adolescentes. Existen riesgos aumentados de problemas asociados a la nutrición, tales como: el exceso de consumo de energía, el consumo elevado de grasa, de colesterol e hidratos de carbono básicos, la desorganización de horarios de alimentación y las comidas fuera del hogar, la deficiencia de nutrientes (calcio), los trastornos de la conducta alimentaria y las adicciones alimentarias (alcohol, cafeína). Se hace hincapié en la necesidad de programas educativos preventivos y del control anual de salud con guía anticipatoria en hábitos alimentarios y actividad física para adolescentes, con el fin de promover hábitos alimentarios saludables hacia la vida adulta. Se revisan en este artículo los principales problemas vigentes en los adolescentes de Chile y América Latina.

(Palabras clave: Nutrición, adolescentes, dietas, adicciones, conducta alimentaria).

Rev Chil Pediatr 2010; 81 (6): 488-497

Trabajo recibido el 30 de abril de 2009, devuelto para corregir el 14 de julio de 2009, segunda versión el 14 de junio de 2010, aceptado para publicación el 02 de diciembre de 2010.

Correspondencia a:

M. Luisa Aguirre Calvo

E-mail: maguirre@med.uchile.cl 


\section{Introducción}

La pubertad y adolescencia se caracterizan por un aumento en la velocidad de crecimiento, cambios en la composición corporal, aparición de caracteres sexuales secundarios y maduración emocional y psicosocial. Estas características influyen tanto en las recomendaciones alimentarias durante esta etapa de la vida, como en los hábitos alimentarios de los adolescentes ${ }^{1}$.

Es frecuente que el adolescente no esté de acuerdo con los patrones familiares de alimentación, incluyendo el intentar adoptar dietas especiales (ej: hacer dieta, dietas vegetarianas) $)^{2,7-9}$.

La autonomía e independencia propias de la edad, se acompañan de un aumento de las actividades fuera del hogar; con horarios de comida desordenados, omisiones de algunos tiempos de alimentación (desayuno, almuerzo) y un aumento de la ingesta de comida rápida, golosinas y bebidas energéticas. Al mismo tiempo, disminuye el consumo de lácteos, frutas y verduras; la cena puede pasar a ser la principal alimentación del día. El sedentarismo, sumado a estos hábitos alimentarios, contribuye a un riesgo elevado de obesidad y otras enfermedades crónicas ${ }^{2-6}$.

Los problemas nutricionales del adolescente, por lo tanto, además de aquellos ocasionados por sobrepeso o desnutrición, se relacionan con malnutrición asociada a malos hábitos y alimentación poco balanceada.

El objetivo de este artículo es actualizar la información disponible en el diagnóstico y tratamiento de los problemas nutricionales prevalentes en los adolescentes chilenos y latinoamericanos, en el contexto de sus hábitos y la sociedad en que viven. Conjuntamente, se revisarán las recomendaciones alimentarias actuales para adolescentes.

\section{Desafíos emergentes en nutrición del adolescente}

\section{Malnutrición por exceso y déficit}

La obesidad es el principal problema nutricional. En Chile, la prevalencia de obesidad en niños menores de 6 años el año 2009 fue de $9,4 \%$, y $22,4 \%$ sobrepeso, según el departamento de estadísticas del Ministerio de salud ${ }^{10}$, cifra que representa al $66 \%$ de la población chilena de esta edad. La prevalencia aumenta con la edad a partir de los 4 años, por lo que en adolescentes se estima que ésta debe ser igual o superior. Por otra parte, es preocupante que el año 2009 , el $15,2 \%$ y $13,7 \%$ de las adolescentes embarazadas menores de 15 años y entre 15 y 19 años respectivamente, presentaron bajo peso, lo que se reduce a menos de la mitad en embarazadas mayores de 20 años ${ }^{10}$.

\section{Deficiencia en consumo de lácteos}

$\mathrm{El}$ adolescente en forma frecuente disminuye el consumo de lácteos, por desconocimiento o por considerarse intolerante a la lactosa (real o subjetivamente), lo que puede llevar a un consumo insuficiente de calcio y riesgo de osteopenia en edades posteriores. Aumenta el consumo de bebidas gaseosas, café, té, y alcohol, los que reemplazan a la leche y los jugos de fruta. La adicción al alcohol u otras sustancias puede comprometer la capacidad de los adolescentes para alimentarse en forma equilibrada, e incluso llevar a una desnutrición ${ }^{5-7}$.

El crecimiento acelerado óseo y muscular durante la adolescencia hace de ésta una etapa crítica en el depósito de calcio en los huesos. Una ingesta adecuada de calcio en la adolescencia ayuda a prevenir fracturas frecuentes en esta edad y disminuye el riesgo de osteoporosis prematura. Con ingestas de calcio menor al $60 \%$ de los requerimientos se compromete la masa ósea, lo que es frecuente en el prepúber y adolescente ${ }^{12,13}$.

La ingesta de lácteos es la principal fuente de aporte de calcio. En un estudio en adolescentes de Estados Unidos que no consumían lácteos, la ingesta de calcio en la dieta era de 480-498 mg/día; y al reformular su dieta sin lácteos en forma balanceada se llegaría a aportes entre 869-1 $160 \mathrm{mg} /$ día, siendo aún insuficiente $^{14}$.

El uso de jugos de frutas fortificados con calcio está en estudio para alcanzar los requerimientos diarios, pero faltan estudios de su biodisponibilidad.

$\mathrm{La}$ actividad física y el deporte son factores 
importantes en la mineralización ósea siendo éste un factor protector en la densidad mineral ósea en la vida adulta ${ }^{15,16}$.

\section{Alteración en la percepción de la Imagen Corporal y asociación con Trastornos de Conducta Alimentaria}

Los adolescentes están altamente influenciados en sus elecciones en alimentación por los medios de comunicación. En las niñas y niños, su imagen corporal es uno de los principales indicadores de logro, por lo que frecuentemente adhieren a dietas restrictivas que les permitan bajar de peso, sumado a un fuerte deseo de imitación de sus pares ${ }^{11,17,18}$.

Los cambios físicos en la figura corporal e insatisfacción con estos cambios, asociado a mayor vulnerabilidad genética o por alteraciones en el aspecto psicosocial del desarrollo de la adolescente, pueden favorecer trastornos de la conducta alimentaria (como anorexia, bulimia o trastornos parciales). Esto es potenciado aún más por la fuerte influencia de los medios de comunicación, que establecen un referente estético con una extrema delgadez ${ }^{11,17,18}$.

Se debe sospechar un Trastorno de la Conducta Alimentaria (TCA) en un (a) adolescente que consulta al médico solo (a) o acompañado (a) por sus padres, preocupado (a) por pérdida o ganancia de peso en un corto período de tiempo y que:

- Adhiere a prácticas poco saludables para control de su peso, asociado a intenso temor a ganar peso (dietas restrictivas, ayuno prolongado, purgas, ejercicio intenso)

- Distorsión en la percepción de su imagen corporal o de su peso

- Trastorno menstrual (oligo o amenorrea)

- Episodios recurrentes de atracones y comportamientos compensatorios para no ganar peso $^{18}$.

\section{Nutrición en el adolescente deportista}

El adolescente con frecuencia viene desarrollando una actividad física previa, la cual puede estar canalizada previamente o se transformará durante la adolescencia en una práctica deportiva, con logros a obtener. Es una inquietud cada vez más frecuente del adolescente y también de los entrenadores e incluso de los familiares, el consumir suplementos nutricionales para mejorar el rendimiento deportivo.

Hay claramente un aumento del gasto energético en proporción a la intensidad del ejercicio $^{19,20}$. Por ejemplo, la natación en estilo libre por 30 minutos para un adolescente de $60 \mathrm{Kg}$ tiene un gasto de $68 \mathrm{Kcal}$; fútbol en torno a 110 Kcal por cada 10 minutos. La evidencia permite afirmar que una alimentación equilibrada, con un buen aporte de macro y micronutrientes, es suficiente para una práctica deportiva intensa. No se justifica, en estas condiciones, el uso de suplementos energéticos, preparados de vitaminas y minerales, para mejorar el rendimiento físico, la musculatura, o la concentración mental ${ }^{21}$. Psicológicamente, su consumo en algunos casos, podría asemejarse a un trastorno de conducta alimentaria.

De todos modos, hay fundamentos para algunos esquemas de consumo de nutrimentos pre-actividad física intensiva, durante una actividad extenuante y posterior a ella. Ellos pueden aportar a mantener una actividad de resistencia en el máximo nivel posible y a lograr una adecuada recuperación post-ejercicio $(\text { tabla } 1)^{21}$.

\section{Suplementos nutricionales en deportistas}

Entre las alternativas que se han propuesto para mejorar el rendimiento deportivo en adolescentes deportistas, está el uso de suplementos de amino-ácidos o concentrados proteínicos, bajo el supuesto que el músculo está compuesto básicamente de agua y proteínas. Estos suplementos proteínicos está hechos en base a leche, huevo, proteína de soya, sin ninguna ventaja sobre el consumo de las fuentes naturales de proteínas, incluidos esos mismos alimentos. Ese exceso potencial de aporte de proteínas traerá aparejado necesariamente un proceso de metabolización, derivando hacia su combustión o hacia el depósito en forma de grasa; el residuo nitrogenado deberá ser eliminado por la orina. Una variante de este aporte aumentado de proteína ha sido el proporcionar fuentes de aminoácidos ramificados, sin un fundamento como para un mejor rendimiento deportivo, una mayor masa muscular o una prevención de la fatiga.

Entre adolescentes principalmente varones 
Tabla 1. Guías de alimentación para adolescentes en deportes de alta exigencia física*

\begin{tabular}{|c|c|c|}
\hline Antes del ejercicio & Durante el ejercicio & Después del ejercicio \\
\hline $\begin{array}{l}\text { Consumir una comida liviana } 3 \mathrm{~h} \text { antes de } \\
\text { la competencia }\end{array}$ & $\begin{array}{l}\text { Reemplazar líquidos según intensidad del } \\
\text { ejercicio y temperatura ambiental }\end{array}$ & $\begin{array}{l}\text { Beber } 500-700 \mathrm{~mL} \text { por c/500 g de peso } \\
\text { perdido por el ejercicio. Evitar bebidas } \\
\text { alcohólicas }\end{array}$ \\
\hline $\begin{array}{l}\text { Beber } 500-700 \mathrm{~mL} \text { de líquidos } 2 \mathrm{~h} \text { antes } \\
\text { de competir }\end{array}$ & $\begin{array}{l}\text { Aportar líquidos 200-400 mL cada 15-20 } \\
\text { min, según tolerancia }\end{array}$ & $\begin{array}{l}\text { Ingerir carbohidratos } 1,5 \mathrm{~g} / \mathrm{Kg} \text { peso en } \\
\text { los primeros } 30 \mathrm{~min} \text {; repetir cada } 2 \mathrm{~h} \text { por } \\
2 \text { ó } 3 \text { veces }\end{array}$ \\
\hline $\begin{array}{l}\text { Evitar alimentos dulces o líquidos previo } \\
\text { a competir }\end{array}$ & $\begin{array}{l}\text { Consumir carbohidratos } 30-60 \mathrm{~g} / \text { hora } \\
\text { de ejercicio (en especial para ejercicios } \\
>1 \mathrm{~h} \text { ) }\end{array}$ & $\begin{array}{l}\text { Después de un ejercicio extenuante: ali- } \\
\text { mentación con proteínas, carbohidratos } \\
\text { y lípidos }\end{array}$ \\
\hline $\begin{array}{l}\text { Si se requiere comida liviana previa, debe } \\
\text { ser alta en carbohidratos (no mono o di- } \\
\text { sacáridos), moderada en proteínas y baja } \\
\text { en grasas y fibra vegetal }\end{array}$ & & \\
\hline
\end{tabular}

*Adaptado de referencia 21.

que participan de actividades deportivas de exigencia física o físicoculturistas comienza a observarse en nuestro país el consumo de productos específicos. Algunos de ellos tienen el carácter de dopaje, con la intención de mejorar el rendimiento deportivo, por ejemplo, los esteroides o los precursores de ellos (androstenediona y dehidroepiandrostenediona DHEA).

La creatina es un compuesto metabólico constituido por glicina, arginina y metioni$\mathrm{na}^{22}$. Ha sido publicitada como una herramienta legal y con mínimos efectos adversos para mejorar masa muscular y es frecuente su promoción y venta en gimnasios y supermercados. Hay evidencia científica que muestra un efecto significativo de aumento de la fuerza muscular asociado a deportes que requieren de una máxima fuerza en un período limitado de tiempo, en condiciones anaeróbicas (ej. lanzamientos en atletismo, 100 metros planos, salto alto, levantamiento de pesas). No tienen efecto en pruebas de resistencia (ej. carreras de larga distancia, ciclismo de ruta). Como efectos adversos se conoce la tendencia a aumentar de peso, calambres musculares, en unos pocos sujetos.

A pesar que es un producto al que no se le ha demostrado una dependencia física o psíquica y está catalogado como un suplemento nutricional, igual tiene como fin el lograr un rendimiento deportivo más allá del logrado por condiciones netamente físicas individuales y por entrenamiento. El Colegio Norteamericano de Deportes sugiere claramente que no debiera usarse en adolescentes menores de 18 años.

\section{Dietas especiales durante la adolescencia}

Como parte del desarrollo de la autonomía y de la identidad propia de los adolescentes, es frecuente que éstos elijan formas de alimentarse que los identifiquen con sus pares o ídolos y los diferencien de su grupo familiar. Las motivaciones son variadas, y las más relatadas son: beneficiar un mejor estado de salud, creencias religiosas diferentes, culturas diferentes en lugares en que existe una alta inmigración, rechazo a consumir alimentos procesados o producidos usando biotecnología, ideales ecológicos ${ }^{23}$.

En EEUU, se sabe que un 2\% de los adolescentes entre 6 y 18 años son vegetarianos, de éstos $0,5 \%$ son veganos o vegetarianos estric$\operatorname{tos}^{24-26}$. No se conoce esta proporción en Chile.

Las dietas que están siendo utilizadas por los adolescentes son, entre otras:

Semivegetarianas: Limitan el consumo de carnes, pero no las excluyen totalmente, pudiendo consumir carne, pollo o pescado, entre 1 vez a la semana a una vez al mes.

Ovolactovegetarianos: Comen preferentemente alimentos de origen vegetal y aceptan comer también huevos, leche y a veces pescado. 
Vegetarianos estrictos o "veganos": Rechazan todos los alimentos de origen animal, sólo consumen alimentos de origen vegetal. Alto riesgo de presentar carencias de nutrientes en adolescentes: vitamina B12, Zn, Fe y ácidos grasos omega 3.

Macrobiótico: A través de la dieta se busca el equilibrio físico y mental, equilibrando las energías yin y yang de los alimentos. Prefieren consumo de cereales integrales, sólo verduras y frutas de la estación, pequeñas porciones de pescado, pollo, queso.

Otras dietas poco presentes en nuestros países incluyen: frutarianas (muy carencial); rastafariana (en general son vegetarianos, y pueden comer pescado).

Las diversas religiones tienen algunas prohibiciones o restricciones alimentarias, que son incorporadas desde la infancia o adolescencia (ej. consumo de alcohol prohibido en musulmanes, adventistas, mormones; consumo de cerdo no consumido por judíos, adventistas, dieta Kosher en judíos, etc. $)^{23}$.

Es importante generar un vínculo adecuado con el adolescente, entendiendo el origen de la elección de su dieta, sin descalificarlo (a), y realizar una adecuada asesoría nutricional.

Una dieta especial puede ser más o menos balanceada según el conocimiento del adolescente y sus padres sobre sus requerimientos, por lo que es fundamental realizar una encuesta nutricional (recordatorio de 24 horas) lo más precisa posible, agregando frecuencia de consumo de alimentos críticos.

Una encuesta alimentaria de frecuencia de consumo en la entrevista nutricional permite evaluar en forma más precisa qué tan adecuada está la ingesta de la mayoría de los nutrientes ${ }^{24}$ :

1. Proteínas: los huevos, lácteos, legumbres, algunos cereales y derivados de la soya (tofu, tempeh, seitan) constituyen buena fuente de aminoácidos esenciales si el aporte calórico a través de otros alimentos es suficiente.

2. Cobalamina (vit B12): Los vegetarianos obtienen esta vitamina de los huevos y lácteos, de alimentos de origen vegetal fortificados con vitamina B12 o de suplementos.

3. Hierro: El hierro "hem" presente en las carnes se absorbe con mayor facilidad; el que se encuentra presente en los vegetales (hierro "no hem") se absorbe mejor si se asocia a una fuente de vitamina C. Los fitatos, presentes en legumbres, nueces y cereales integrales, también disminuyen parcialmente la absorción de hierro. En Chile y otros países latinoamericanos, la fortificación con Fe de la harina de trigo permite tener un aporte básico aceptable de Fe.

4. Zinc: La absorción de zinc también está inhibido por los fitatos contenidos naturalmente en legumbres, semillas (nueces, almendras) y cereales naturales o integrales.

5. Calcio: Los lácteos son buena fuente de $\mathrm{Ca}$ (200 a $250 \mathrm{mg} /$ porción), y si éstos no están permitidos, existen derivados de la soya fortificados con calcio (tofu, jugos, lácteos, queso, yogurt) que permitirían cubrir los requerimientos de este mineral. Otras fuentes vegetales de calcio requieren ingerir gran cantidad de ésta para alcanzar lo aportado por un vaso de leche.

6. Vitamina D: La exposición suficiente a luz solar previene la deficiencia de vitamina $\mathrm{D}$; puede ser un factor de riesgo la baja exposición solar incluso para adolescentes, si viven en zonas muy australes de Chile y Argentina $^{27}$. Si el adolescente no consume lácteos fortificados con vitamina $\mathrm{D}$, puede obtenerla de otros alimentos de origen vegetal fortificados con vitamina D.

7. Ácidos grasos omega 3: Si la dieta especial no permite la ingestión de pescado, huevos o grandes cantidades de algas marinas, es probable una deficiencia de ácidos grasos omega 3. Otras fuentes de ácido $\alpha$-linolénico son: aceites en base a pescado, el aceite de canola, soya, tofu, nueces, semilla de Chia, linaza.

Se recomienda mantener adolescentes vegetarianos en control y seguimiento en centros de atención de adolescentes, para asegurar en ellos una adecuada supervisión de salud, considerando el alto riesgo de desarrollar carencias nutricionales específicas y/o ser ésta una manifestación de un trastorno de conducta alimentaria oculto. Los criterios de derivación a un equipo especializado de nutrición incluyen: adolescente vegetariano estricto, frutariano o 
vegano; adolescente muy restrictivo en su alimentación, con bajo peso o retraso en curva de crecimiento, que no adhiere a indicaciones nutricionales a nivel primario; adolescente vegetariano con trastorno de conducta alimentaria; embarazada adolescente vegetariana.

\section{Adicciones en el adolescente y nutrición}

Entre las adicciones asociadas a la nutrición del adolescente están principalmente el consumo de cafeína y de alcohol. La cafeína se obtiene directamente del café, té, o a través del consumo de gaseosas. La concentración de cafeína fluctúa entre $100 \mathrm{mg}$ para una taza de café molido corriente (180 mL); 45-65 mg para una gaseosa individual de $360 \mathrm{~mL}$, o $80-120$ $\mathrm{mg} /$ porción para las bebidas altas en cafeína disponibles en el mercado chileno (SERNAC, tabla 2).

Estudios en adultos muestran que la adicción es posible de observar con consumo promedio diario moderadamente elevado $(235 \mathrm{mg}$ de cafeína), es decir, 2,5 tazas de café molido ${ }^{28}$. Los signos de dependencia ante la suspensión del café son principalmente: cefalea moderada a intensa, depresión, ansiedad, uso no prescrito de otros medicamentos ${ }^{28}$. Un estudio más reciente en adolescentes muestra que el alto consumo de cafeína se asocia con dificultades para dormir y sensación de cansancio en las mañanas $^{29}$. El efecto parece ser consistente aunque de una magnitud leve y sin conocerse su impacto a largo plazo. Las evidencias actuales respecto del consumo de café, no permiten

Tabla 2. Contenido de cafeína en alimentos y bebidas

\begin{tabular}{|llc|}
\hline Alimento & Porción & $\begin{array}{c}\text { Cafeína } \\
\text { (mg/porción) }\end{array}$ \\
\hline Café instantáneo & $180 \mathrm{~mL}$ (taza) & $60-70$ \\
Café Capuccino & $180 \mathrm{~mL}$ (taza) & 70 \\
Café molido & $180 \mathrm{~mL}$ (taza) & $100-250$ \\
Yerba mate & $180 \mathrm{~mL}$ (taza) & $10-60$ \\
Barra de chocolate & Barra & $60-70$ \\
Coca Cola & $360 \mathrm{~mL}$ (lata) & 65 \\
\hline Pepsi Cola & $360 \mathrm{~mL}$ (lata) & 45 \\
\hline Bebidas energéticas & $250 \mathrm{~mL}$ (lata) & $80-125$ \\
\hline
\end{tabular}

sugerir una exclusión para adolescentes, pero sí un consumo moderado, por debajo de las cantidades asociadas a riesgo de dependencia y de efectos adversos.

El alcohol es otro componente de la alimentación cuyo consumo comienza en la adolescencia, asociado a fiestas y reuniones grupales con sus pares; sin embargo, puede avanzar hacia un alcoholismo hacia el final de la adolescencia, con dependencia tanto física como psíquica, en especial en los grupos sociales más deprivados. Contribuye además a un aporte excedido de energía y depósito de grasa corporal ${ }^{30}$.

Aunque no están asociadas directamente a la nutrición, otras adicciones como el cigarrillo y la marihuana, pueden tener efectos sobre la alimentación o la metabolización de nutrientes. Un estudio reciente en adultos jóvenes norteamericanos corroboraba que el consumo regular de marihuana estaba asociado a: sexo masculino, un mayor consumo de energía y de alcohol, sin asociación con alteraciones de presión arterial, lípidos séricos, glicemia o $\mathrm{IMC}^{31}$.

Respecto al consumo de cigarrillos se ha observado en adolescentes norteamericanos una asociación con menor consumo de lácteos y vegetales, así como menor cantidad de actividad física ${ }^{32}$. También parece tener asociación con un riesgo aumentado de síndrome metabólico, tanto en los adolescentes que fuman (OR 6,1; IC: 2,8-13,4) como en aquellos expuestos a cigarrillo ambiental (OR 4,7; IC: 1,7-12,9). Este efecto fue observado tanto en adolescentes no obesos y obesos ${ }^{33}$.

\section{Recomendaciones alimentarias para adolescentes}

La alimentación debe cubrir las necesidades de energía y nutrientes, considerando la edad, el género y el nivel de actividad física. En especial debe considerarse este último factor ya que a igual edad, un niño practicando deportes de alta demanda de energía puede incluso duplicar sus requerimientos en relación a un niño sedentario. Se recomienda en estas situaciones mantener una proporción adecuada de nutrientes, en torno a un $15 \%$ de la energía 
como proteínas de origen animal y vegetal, 20 a $30 \%$ como grasas y 55 a $60 \%$ como carbohidratos.

Energía: Para estimar la recomendación de ingesta energética en los adolescentes, es necesario considerar la actividad física de ellos, su edad y género ${ }^{1}$. En Chile, las recomendaciones de ingesta energética para adolescentes, sólo consideran un grupo de edad y actividad física moderada, lo que en la mayoría de los casos (adolescentes sedentarios), se encuentran por sobre su requerimiento, favoreciendo el aumento de peso y la obesidad. Desde el año 2001, disponemos de nuevas recomendaciones de energía por la $\mathrm{FAO} / \mathrm{OMS}^{34}$, las que pueden ser estimadas con las siguientes fórmulas que consideran edad, sexo y actividad física, o utilizar los valores descritos en la tabla 3.

Requerimiento energético hombres 9 a 18 años $(\mathrm{Kcal} /$ día $)=113,5-61,9 *$ edad $($ años $)+$ $\operatorname{AF} *(26,7 *$ peso $(\mathrm{kg})+903 *$ talla (metros)).

Requerimiento energético mujeres 9 a 18 años $(\mathrm{Kcal} /$ día $)=160,3-30,8^{*}$ edad (años $)+$ $\operatorname{AF}^{*}(10 *$ peso $(\mathrm{kg})+934 *$ talla (metros)).

Coeficientes de actividad física (AF): para varones: 1,0 sedentarios; 1,13 levemente activos; 1,26 activos y 1,42 muy activos. Para mujeres: 1,0 sedentarias; 1,16 levemente activas; 1,31 activas y 1,56 muy activas.

Proteínas: Las necesidades de proteínas para adolescentes varían de acuerdo al desarrollo puberal y patrón de crecimiento. El nivel seguro de ingesta de proteínas (NSI) de acuerdo a las últimas recomendaciones año 2002, es $0,95 \mathrm{~g} / \mathrm{kg} /$ día para adolescentes entre 11 y 14 años, y de $0,8 \mathrm{~g} / \mathrm{kg} /$ día para adolescentes entre 15 y 18 años. Se puede observar un consumo inadecuado de proteínas en adolescentes que siguen una dieta vegetariana estricta y no balanceada (veganos), en los trastornos de conducta alimentaria, en enfermedades crónicas y embarazo ${ }^{35}$.

Hidratos de carbono: Los hidratos de carbonos simples no debieran aportar más del $10 \%$ del total de carbohidratos, el resto $(90 \%)$ deben ser de predominio almidones. La mitad de los cereales deben aportarse como cereales integrales, para contribuir a alcanzar la recomendación de fibra dietaria.

Grasas: La proporción de grasas saturadas, poli-insaturadas y mono-insaturadas debiera ser de 1:1:1, con énfasis en el aporte de los ácidos grasos mono-insaturados (aceite de oliva, palta) y de los omega 3 (productos y aceites marinos) $)^{1,36}$.

Micronutrientes: Las necesidades de minerales aumentan durante el crecimiento acelerado propio de la adolescencia. Durante esta época adquieren especial importancia el calcio, hierro y zinc, por su rol en crecimiento y por su ingesta potencialmente inadecuada.

Calcio: Los requerimientos para hombres y mujeres adolescentes es de $1300 \mathrm{mg} /$ día como ya enunciamos, existiendo una gran dificultad para alcanzarlos si no hay un consumo adecuado de lácteos.

Hierro: Aumentan el requerimiento de hierro $(\mathrm{Fe})$ a esta edad, el incremento en la masa muscular y volumen sanguíneo propio del desarrollo puberal en varones y las pérdidas aumentadas por el sangramiento menstrual en mujeres. La recomendación de ingesta de Fe

Tabla 3. Recomendaciones de ingesta energética (Kcal/día) para adolescentes, según edad, género y nivel de actividad física. (adaptado de referencia 1, 36)

\begin{tabular}{|lcccc|}
\hline Género & Edad (años) & Actividad física (AF) & \\
Mujer & & Sedentario* & Moderada** & Activo*** \\
& $9-13$ & 1600 & $1600-2000$ & $1800-2200$ \\
Hombre & $14-18$ & 1800 & 2000 & 2400 \\
& $9-13$ & 1800 & $1800-2200$ & $2400-2600$ \\
\hline
\end{tabular}

*sedentario se refiere a la actividad física habitual de la vida diaria. ${ }^{* *} \mathrm{AF}$ moderada se refiere al equivalente a caminar rápido 2 a $3 \mathrm{~km} /$ día, además de las actividades habituales de la vida diaria. ${ }^{* * *}$ Activo se refiere a aquel que practica deportes por varias horas, varias veces a la semana, o aquel que se moviliza siempre caminando o en bicicleta, o aquel que realiza actividades diarias que requieren mucha energía (cargador portuario, trabajador agrícola, etc). 
en adolescentes entre 9 y 13 años es de $8 \mathrm{mg} /$ día en hombres y mujeres y aumenta a $11 \mathrm{mg} /$ día en hombres y $15 \mathrm{mg} /$ día en mujeres entre 14 y 18 años $^{37}$. La concentración de $\mathrm{Fe}$ en una dieta habitual es de $6 \mathrm{mg} / 1000 \mathrm{kcal}$, por lo que las adolescentes que ingieren menos de 2000 $\mathrm{kcal} /$ día pueden no alcanzar el aporte suficiente de Fe.

Zinc: El Zn es especialmente importante durante la adolescencia por su rol en crecimiento y maduración sexual. La ingesta recomendada para hombres y mujeres adolescentes entre 9 y 13 años es de $8 \mathrm{mg} /$ día, y entre 14 y 18 años aumenta a $11 \mathrm{mg} /$ día en hombres y 9 $\mathrm{mg} /$ día en mujeres, asumiendo una dieta con una adecuada biodisponibilidad de $\mathrm{Zn}$ (consumo bajo de fitatos) ${ }^{37}$.

Vitaminas: Los requerimientos de vitaminas también aumentan durante la adolescencia. Las vitaminas A y $\mathrm{C}$ son importantes para el crecimiento de nuevas células y piel sana. La vitamina $\mathrm{D}$ se requiere especialmente para el rápido crecimiento óseo, y la recomendación de ingesta para hombres y mujeres entre 9 y 18 años, según las últimas recomendaciones de la Academia Americana de Pediatría en 2008, es de $10 \mu \mathrm{g} /$ día (400 UI), a través del uso de alimentos fortificados y adecuada exposición solar $^{37}$.

Ácido fólico: Se recomienda un aumento en la ingesta de ácido fólico a $400 \mu \mathrm{g} / \mathrm{día}$, especialmente en mujeres adolescentes en edad fértil, porque una deficiencia de folato en el primer trimestre del embarazo se correlaciona con defectos en el desarrollo del tubo neural del feto, recomendación que en Chile se alcanza gracias a la fortificación de la harina e ingesta suficiente de pan y fideos ${ }^{38}$.

\section{Hábitos alimentarios}

Para compatibilizar los requerimientos de nutrientes con los aspectos psicológicos y sociales asociados, la alimentación debe ser variada y atractiva. El desayuno es importante para el adolescente, pues le ofrece energía y nutrientes necesarios para sus actividades matutinas, en particular su rendimiento escolar. Hay discusión acerca del efecto de la ausencia de desayuno en el rendimiento escolar. Algunos estudios han sugerido que en niños que frecuentemente asisten al colegio sin haber ingerido desayuno puede verse afectado el rendimiento escolar, por presentar dificultades de atención, mayor inquietud y en algunos menor capacidad de abstracción matemática, sin embargo otros estudios no lo demuestran. Se requieren, por lo tanto, estudios con métodos más específicos para concluir definitivamente si hay una asociación en tal sentido y controlando por otros posibles factores confundentes $^{39,40}$.

Las colaciones deben ser en base a alimentos saludables, evitando la incorporación de alimentos industrializados como golosinas. Ejemplos de alimentación saludable es fruta, vegetales, lácteos descremados, frutos secos y/o semillas.

Deben establecerse horarios de alimentación, pero no rígidos, adaptándolos a las actividades escolares y familiares, evitando el consumo de alimentos fuera de horario.

Se recomienda mantener limitado el tamaño de las porciones y reducir la ingesta de energía en las comidas nocturnas. También evitar comer frente al televisor, computador o videojuegos. El comer en familia es protector para lograr hábitos saludables de alimentación y disminuir otras conductas de riesgo ${ }^{7}$.

\section{Guía para la alimentación de adolescentes}

De acuerdo a la guía alimentaria de EEUU del año $2006^{36}$, que considera los nuevos requerimientos de energía por edad, el incremento de la obesidad y el sedentarismo a toda edad, se recomienda lo siguiente:

1. Los adolescentes deben realizar al menos 1 $\mathrm{h}$ de ejercicio moderado todos los días de la semana y limitar horario de actividades sedentarias como ver TV o sentarse frente a computador o video juegos a 1 a $2 \mathrm{~h}$ al día máximo.

2. El consumo de lácteos en adolescentes debe ser el equivalente a 4 tazas de leche descremada al día.

3. El adolescente debe consumir al menos 2 tazas de fruta al día, y 2 y media tazas de verdura al día, de grupos y colores variados durante la semana. 
4. Incorporar 5 a 7 porciones al día de alimentos del grupo de los cereales según edad y nivel de actividad física (una porción equivale a media taza de arroz, fideos, papas, choclo, cereales, maíz, o medio pan o 1 tostada de pan de molde), de los cuales el 50\% debe corresponder a cereales integrales.

5. El 25 a $35 \%$ de las calorías totales deben provenir de grasas, favoreciendo el consumo de pescado, nueces y aceites de origen vegetal. Limitar consumo de colesterol a no más de $300 \mathrm{mg} /$ día, grasas saturadas a no más del $10 \%$ de las calorías totales, disminuir al máximo posible el consumo de ácidos grasos trans.

6. Consumir menos de $2300 \mathrm{mg}$ de sodio al día, y asegurar ingesta de potasio de 4700 $\mathrm{mg} /$ día a través de alimentos.

7. Los adolescentes deben consumir carne, pavo, pescado o pollo con bajo contenido de grasas, una porción 2 veces a la semana, legumbres 1 taza 2 veces a la semana, huevo 1 unidad 2 a 3 veces a la semana.

8. Los adolescentes no deben consumir alcohol.

9. Los adolescentes no deben consumir alimentos que contengan leche no pasteurizada, huevos o pescados o mariscos parcialmente cocidos.

En resumen, los aspectos nutricionales durante la adolescencia incluyen problemas variados, algunos propios de estas edades y otros que adquieren una especial relevancia en esta etapa: obesidad, trastornos de la conducta alimentaria, carencias nutricionales específicas, adicciones asociadas a la alimentación. Se requiere una visión preventiva por los equipos de salud desde edades previas, durante la adolescencia con sus controles de salud anuales, y una derivación oportuna a los grupos profesionales especializados.

\section{Referencias}

1.- American Heart Association, Giding SS, Dennison BA, Birch LL, et al: Dietary recommendations for children and adolescents: A guide for practitioners. Pediatrics 2006; 117: 544-59.

2.- Larson NI, Story M, Bsenberg ME, Neumark-Sztaner D:
Food preparation and purchasing among adolescents: Associations with sociodemographic characteristics and diet quality. J Am Diet Assoc 2006; 106: 211-8.

3.- Peerson N, Biddle SJ, Gorely T: Family correlates of fruit and vegetable consumption in children and adolescents: a systematic review. Public Health Nutr 2009; 12: 267-83.

4.- Ebbeling CB, Sincalir KB, Garcia-Lago E, Feldman $H A$, Ludwig DS: Compensation for energy intake form last fast food among overweight and lean adolescents. JAMA 2004; 291: 2828-33.

5.- Sjöberg A, Hallberg L, Höglund D, Hulthén L: Meal pattern, food choice, nutrient intake and lifestyle factors in the Göteborg adolescence study. Eur J Clin Nutr 2003; 57: 1569-78.

6.- Niemeier H, Raynor H, Lloyd-Richardson E, Rogers $M$, Wing $R$ : Fast food consumption and breakfast skipping: Predictors of weight gain from adolescence to adulthood in a nationally representative sample. J Adolesc Health 2006; 39: 842-9.

7.- Larson NI, Neumark-Sztainer D, Hannan P, Story M: Family meals during adolescence are associated with higher diet quality and healthful meal patterns during young adulthood. J Am Diet Assoc 2007; 107: 1502-10.

8.- Vicente-Rodriguez G, Rey-López JP, Martín-Matillas M, et al; AVENA Study Group: Television watching, videogames, and excess of body fat in Spanish adolescents: the AVENA study. Nutrition 2008; 24: 654-62.

9. Haerens L, Craeynest M, Deforche B, Maes L, Cardon $G$, De Bourdeaudhuij I: The contribution of psychosocial and home environmental factors in explaining eating behaviours in adolescents. Eur J Clin Nutr 2008; 62: 51-9.

10.- DEIS: Ministerio de Salud de Chile. www.minsal.cl

11.- Taylor CB, Sharpe T, Shisslak C, et al: Factors associated with weight concerns in adolescent girls. Int J Eat Disorders 1998; 24 (1): 31-42.

12.- Loud KJ, Gordon CM: Adolescent bone health. Arch Pediatr Adolesc 2006; 160: 1026-32.

13.- Lonou AJ, Berkow SE: Calcium, dairy products and bone health in children and young adults: a reevaluation of the evidence. Pediatrics 2005; 115: 736-43.

14.- Gao X, Wilde PE, Lichtenstein AH, Tucker KL: Meeting adequate intake for dietary calcium without dairy foods in adolescent aged 9 to 18 years (NHNES 2001-2002). J Am Diet Assoc 2006; 106: 1759-65.

15.- Lloyd T, Chinchilli VM, Johnson-Rollings $N$, et al: Adult female hip bone density reflects teenage sports-exercise pattern but not teenage calcium intake. Pediatrics 2000; 106: 40-4. 
16.- Ondrak KS, McMurray RG: Physical activity, calcium intake and bone health in children and adolescents. Sports Med 2007; 37: 587-600.

17.- Hill AJ: Motivation for eating behaviour in adolescent girls: the body beautiful. Proc Nutr Soc 2006; 65: 37684.

18.- Chamay-Weber C, Narring F, Michaud PA: Partial eating disorders among adolescents: a review. J Adolesc Health 2005; 37: 417-27.

19.- Roemmich JN, Richmond RJ, Rogol AD: Consequences of sport training during puberty. J Endocrinol Invest 2001; 24: 708-15.

20.- Petrie HJ, Stover EA, Horswill CA: Nutritional concerns for the child and adolescent competitor. Nutrition 2004; 20: 620-31.

21.- Position of the American Dietetic Association, Dietitians of Canada, and the American College of Sports Medicine: Nutrition and Athletic Performance. J Am Diet Assoc 2000; 100: 1543-56.

22.- Kreider $R B$ : Effects of creatine supplementation on performance and training adaptations. Mol Cell Biochem 2003; 244: 89-94.

23.- American Dietetic Association: Cultural Competence Overview. En: Pediatric Manual of Clinical Dietetics, Nevin-Folino NL editor, second edition, 2008, p. 3-18.

24.- Herbold NH, Frates SE: Update of nutrition guidelines for the teen: trends and concerns. Curr Opin Pediatr 2000; 12: 303-9.

25.- Nutritional Aspects of Vegetarian Diets. En: Pediatric Nutrition Handbook, $6^{\text {th }}$ edition. American Academy of Pediatrics. Kleinman RE ed. 2009, p. 201-19.

26.- American Dietetic Association: Nutrition for the vegetarian child. En: Pediatric Manual of Clinical Dietetics, Nevin-Folino NL editor, second edition, 2008, p. 99108.

27.- Wagner CL, Greer FR and the Section on breastfeeding and committee on nutrition: Prevention of rickets and vitamin D deficiency in infants, children and adolescents. Pediatrics 2008; 122: 1142-52.

28.- Silverman K, Evans SM, Strain EC, Griffiths RR: Withdrawal syndrome after double-blind cessation of caffeine consumption. New Engl J Med 1992; 327: 1109-14.
29.- Orbeta $R$, Overpeck $M D$, Ramcharran D, Kogan $M D$, Ledsky $R$ : High caffeine intake in adolescents: associations with difficulty sleeping and feeling tired in the morning. J Adolesc Health 2006; 38: 451-3.

30.- Caton SJ, Marks JE, Hetherington MM: Pleasure and alcohol: Manipulating pleasantness and the acute effects of alcohol on food intake. Physiol Behav 2005; 84: 3717.

31.- Rodondi N, Pletcher MJ, Liu K, Hulley SB, Sidney S: Marijuana use, body mass index, and cardiovascular risk factors (from the CARDIA Study). Am J Cardiol 2006, 98: 478-84.

32.- Wilson DB, Smith BN, Speizer IS, et al: Differences in food intake and exercise by smoking status in adolescents. Prev Med 2005; 40: 872-9.

33.- Weitzman $M$, Cook $S$, Auinger $P$, et al: Tobacco smoke exposure is associated with the metabolic syndrome in adolescents. Circulation 2005; 112: 862-9.

34.- FAO/WHO/UNU: Expert Consultation. Human energy requirements. Rome. World Health Organization 2004.

35.- WHO/FAO/UNU: Expert consultation. Protein requirements. Geneva, World Health Organization 2005.

36.- Dietary Guidelines for Americans 2005: US Department of Health and Human Services. US Department of Agriculture. www.healthierus.gov/dietary guidelines

37.- Dietary Reference Intakes Recommended Intakes for Individuals: Food and Nutrition Board, Institute of Medicine. http://www.iom.edu/Reports/2006/Dietary-Reference-Intakes-Essential-Guide-Nutrient-Requirements. aspx

38.- Evidencias del éxito del programa de fortificación de las harinas. Eva Hertrampf. www.redsalud.gov.cl/portal/url/ page $/ \mathrm{minsal.cl} / \mathrm{g}$ _proteccion/g_alimentos/prot_fortificacion.html

39.- Kleinman R, Hall S, Green H, et al: Diet, breakfast and academic performance in children. Ann Nutr Metab 2002; 46: 24-30.

40.- Ells LJ, Hillier FC, Shucksmith J, et al: A systematic review of the effect of dietary exposure that could be achieved through normal dietary intake on learning and performance of school-aged children of relevance to UK schools. Br J Nutr 2008; 100: 927-36. 